\title{
Cell division zones are maintained by the photoassimilate-auxin-brassinosteroid-polar auxin transport signal relay in Arabidopsis root tips
}

\author{
Jun Sakaguchi ${ }^{1}$ and Yuichiro Watanabe ${ }^{1}$ \\ ${ }^{1}$ Tokyo Daigaku Daigakuin Sogo Bunka Kenkyuka Kyoyo Gakubu Seimei Kankyo \\ Kagakukei
}

February 8, 2022

\begin{abstract}
Light-derived signals from the shoot tissue promote root growth. Brassinosteroids (BRs), which are phytohormones, are crucial for root growth and leaf expansion. We previously revealed that photoreceptor-dependent signals promote the expression of DWARF 4 ( $D W F 4$ ), which encodes a key BR biosynthesis-related enzyme, in the root tips. Furthermore, auxin is indispensable for root growth. In this study, we focused on the relationships among light-derived signals, auxin, and BR in the root tips. Polar auxin transport (PAT) in the root tips was undetectable in plants grown on medium lacking sucrose for $24 \mathrm{~h}$ in darkness; however, a BR treatment restored PAT in darkness. We also observed that PAT was non-existent in the cell division zones under BR-deficient conditions, even under light. On the basis of these results, we propose that the light-dependent cooperative effects of auxin and BR in the root tip cell division zone are necessary for sustaining root growth.
\end{abstract}

\section{Introduction}

Light, temperature, water availability, and soil nutrient contents are important environmental factors affecting the growth of land plants. Additionally, coordinated responses to environmental and intrinsic signals, some of which are mediated by phytohormones, are important for plants to sense and adapt to fluctuating external environments. Light, which is a critical environmental signal, is a source of energy for photosynthesis, while also a determinant of plant morphogenesis patterns. In photomorphogenesis or skotomorphogenesis, brassinosteroid (BR) and auxin coordinately mediate responses to environmental signals (Maharjan et al. , 2014; Tian et al. , 2018; Sun et al. , 2020). The growth of aboveground and belowground plant parts is coordinated; however, the underlying molecular mechanism and how BR and auxin are involved are still largely unknown.

Arabidopsis has several BR biosynthesis pathways, but the major ones are the early and late C-6 oxidation pathways (Choe et al. , 1998; Noguchi et al. , 2000; Fujioka, Takatsuto and Yoshida, 2002; Kim et al. , 2005; Fujita et al. , 2006; Ohnishi et al. , 2006). Moreover, DWARF4 (DWF4), which is a $22 \alpha$ hydroxylase that belongs to the CYP90 family, catalyzes the rate-limiting step of the predominant BR biosynthesis pathway (Choe et al. , 1998; Shimadaet al. , 2003; Fujita et al. , 2006). Similar to other BR-deficient mutants, the hypocotyl of the $d w f 4$ mutant essentially does not elongate in darkness (Li and Chory, 1997; Azpirozet al. , 1998; Choe et al. , 1998; Li et al. , 2001; Kim et al. , 2005). We previously observed that the mutant roots are as long as the wild-type (WT) roots when plants are incubated in darkness, but they are much shorter than the WT roots when plants are exposed to continuous light (Sakaguchi and Watanabe, 2017). We established $D W F 4$-GUS plants, in which DWF 4 expression can be monitored according to the expression of the marker gene encoding beta-glucuronidase (GUS) in the dwf4-102 mutant background. Analyses of the DWF4-GUS plants indicated that light perception mainly through the blue light photoreceptor CRYPTOCHROME 
(CRY1) in shoots induces DWF4 accumulation in root tips, leading to enhanced root growth (Sakaguchi and Watanabe, 2017; Sakaguchi, Matsushita and Watanabe, 2019). Interestingly, in darkness, DWF 4 expression was maintained at a certain level in the root cell division zone, but not in the root cell elongation zone. The similarity in the root growth of WT and dwf4-102 mutant plants in darkness compelled us to study the coordination between the light-dependent signaling in shoots and the BR biosynthesis pathway in the root cell division zone during the maintenance of constant or minimal root tip growth.

Brassinosteroid and auxin signaling pathways are coordinated to mediate responses to environmental signals. The objective of this study was to identify the presumptive signal relay involving BR, auxin, and light signals on the basis of our results and those of recent studies to explain meristem maintenance and root growth under light and dark conditions.

\section{Results}

$D W F 4$ expression in the cell elongation zone but not the cell division zone depends on the photoreceptors-derived signals.

Plants respond to various signals from their circumstance and the environmental information often transmitted from the perceived tissue to the others. Many studies conducted grafting experiments to reveal the long-distance signals to elucidate the communications between the shoot tissue and the root (Osugi et al., 2017; Ha et al., 2018; Ota et al., 2020). Our earlier work indicated that the light perception in the shoot tissue contributed to the DWF 4 expression in the root tip and subsequent root growth (Sakaguchi and Watanabe, 2017). Furthermore, we indicated that the DWF4 expression in the cell elongation zone in the primary root tip depends on the photoreceptors (Sakaguchi, Matsushita and Watanabe, 2019). However, photoreceptors-derived signals seemed to have less impact on the DWF 4 expression in the cell division zone. To confirm whether the photoreceptors-derived signals contribute to the $D W F 4$ expression in the cell division zone, we performed micrografting between photoreceptors mutant seedlings and $D W F$ - $G U S$ seedlings. Here, we focused on CRY1 and the red/far-red light photoreceptor PHYTOCHROME B (PHYB) because the double mutantscry1phyB indicated the retarded root growth in the continuous light and the decrease of the DWF 4 expression level in the root tips (Sakaguchi, Matsushita and Watanabe, 2019). The grafts with the cry1phyB scion showed retarded root growth (Fig. 1a). This result indicated that photoreceptorsderived signals from the shoots promote root growth (Ha et al. , 2018; Sakaguchi, Matsushita and Watanabe, 2019). We observed DWF4-GUS accumulation in the cell division and elongation zone in the primary root when the WT scions were grafted onto the DWF4-GUS rootstocks. However, DWF4-GUS accumulation was observed in the cell division zone but not the cell elongation zone whencry 1 phy $B$ scions grafted onto $D W F 4-G U S$ rootstocks (Fig. 1b, c). These results indicated that the photoreceptors-derived signals from the shoot tissue but not in the root tips are indispensable for $D W F 4$ expression in the cell elongation zone and that $D W F 4$ expression in the cell division zone depends on the other light-dependent signals. Next, we performed micrografting between cry1phyB seedlings and PIN-FORMED1(PIN1 )-GFP seedling to observe the auxin flow in the cell division zone. We observed the plasma membrane (PM) localized PIN1-GFP in the stele when the WT and cry1phyB scions were grafted ontoPIN1-GFP rootstocks (Fig. 1d, e). These results indicated that photoreceptors in the shoot tissue have less impact on the auxin flow in the cell division zone. Integrating the results of the grafting experiments, the light-dependent signals except for the photoreceptors contribute to the auxin and BR in the cell division zone, although the size of the cell division zone decreased when cry1phyB scions were grafted.

\section{Localized auxin synthesis in the cell division zone enhanced DWF4 expression}

In a previous study, we demonstrated that growth on sucrose-minus medium in darkness results in a lack of DWF4 accumulation in the cell division zone, but an exogenous auxin treatment quickly restores DWF4 levels (Sakaguchi and Watanabe, 2017). These findings suggest auxin is synthesized locally in root tips, thereby influencing cell division and $D W F 4$ expression in the cell division zone. Regarding auxin biosynthesis, the indole-3-pyruvic acid pathway is widely conserved in land plants. Moreover, it is catalyzed by TRYPTOPHAN AMINOTRANSFERASE OF ARABIDOPSIS1 (TAA1) and YUCCA (YUC) enzymes in 
Arabidopsis (Zhaoet al. , 2001; Stepanova et al. , 2008; Sugawara et al. , 2009; Yamada et al. , 2009; Mashiguchi et al. , 2011). The Arabidopsis genome includes 11 YUC-encoding genes, each of which has a distinct expression pattern (Cheng, Dai and Zhao, 2006; Chenet al. , 2014). Five YUC genes (YUC3/5 $/ 7 / 8 / 9$ ) are highly expressed in root tips and affect root growth (Chen et al. , 2014; Matosevich et al. , 2020). The yис3уис5yис7уис8уис9( уисQ ) quintuple mutant exhibits severely retarded root growth, but its shoot growth is relatively unaffected and the overproduction of auxin in the shoot tissue cannot restore root growth (Chen et al. , 2014).

We first examined the effects of an auxin biosynthesis inhibitor on $D W F 4$ expression and root growth. We selected the TAA1 inhibitor L-kynurenine (Kyn) (He et al. , 2011). We expected that the inhibition of TAA1, which acts upstream of YUCs, would substantially decrease auxin biosynthesis, even if the lightdependent signals promoted $Y U C$ gene expression. The $D W F 4-G U S$ plants were grown on sucrose-minus medium in plates under continuous light until 6 days after germination (DAG). They were then transferred to plates containing agar medium with or without Kyn and incubated under the same light condition for 24 h. Compared with the mock treatment, the Kyn treatment suppressed DWF4-GUS accumulation in the cell division zone in a dose-dependent manner (Fig. 1a-d). Similarly, the DWF4-GUS content in the cell division zone decreased and was restricted to the apex after a 24-h incubation in darkness (Fig. 1e). Accordingly, localized auxin biosynthesis in the cell division zone appears to modulate $D W F$ 4expression and its effects may depend on light. Next, an examination of root growth revealed that Kyn had detrimental effects (Fig. 1f). These results suggested that a decrease in the auxin content and the subsequent decrease in the BR content in the cell division zone led to retarded root growth.

Because $D W F 4$ expression in the cell division zone appeared to depend on the auxin supply, we investigated whether the five YUCs indispensable for auxin biosynthesis in the root tip zone influenced $D W F 4$ expression. A quantitative real-time polymerase chain reaction (qRT-PCR) analysis of $D W F 4$ expression in a 5-mm region of the primary root tips revealed a decrease in expression in theyucQ mutant (Fig. $1 \mathrm{~g}$ ). Considered together, these findings implied that $D W F 4$ expression in the cell division zone depended on auxin production via the TAA1-YUC biosynthetic pathway.

\section{Some $Y U C$ genes were expressed in the root tips in darkness}

We then analyzed which YUC enzymes affect DWF4 expression in the cell division zone by examining the root tips of transgenic plants expressing the GUS marker gene under the control of the YUC3, 7,8 , or 9 promoter under continuous light or in darkness (Fig. 2a-h). In the proYUC3-GUS ,proYUC7-GUS, and proYUC9-GUS plants, the GUS signal was detected in the cell division zones under continuous light and in continuous darkness, suggesting that some YUC enzymes are present in root tips even in the absence of light. The GUS signal was also detected in proYUC8-GUS plants in darkness, which differed somewhat from the results of an earlier study (Chen et al. , 2014) (Fig. 2i, j). These observations indicated that a certain amount of auxin is synthesized in root tips irrespective of light conditions.

We speculated that $Y U C$ expression, which reflects auxin biosynthesis in root tips, is maintained in darkness, but is enhanced when plants are exposed to light. This localized auxin supply induces $D W F 4$ expression in the cell division zone. Next, we addressed whether photoassimilates function as light-dependent signals contributing to auxin biosynthesis in root tips.

\section{Sugar or photoassimilates promoted auxin biosynthesis by YUC7/9 in root tips}

Photoassimilates are transported throughout plant tissues as sucrose, but trigger various signals as glucose (Rolland, Moore and Sheen, 2002; Moore, 2003). An earlier study confirmed that sucrose functions as a longdistance signal that controls root growth (Kircher and Schopfer, 2012). Another study demonstrated that photoassimilates increase auxin biosynthesis by inducing the expression of several $Y U C$ genes (Sairanen et al. , 2013). Thus, we tested whether photoassimilates (e.g., sucrose or glucose) can enhance auxin metabolism in root tips in darkness. We grew proYUC3/7/8/9-GUS plants on medium containing $1 \%$ sucrose for 6 days in darkness. Plants were divided into groups, with one group transferred to $1 \%$ sucrose medium and another group transferred to medium lacking sucrose/glucose. Plants were incubated for $24 \mathrm{~h}$ in darkness. One of the 
groups on the medium lacking sucrose/glucose was transferred to $1 \%$ glucose medium and incubated for $3 \mathrm{~h}$ in darkness (i.e., glucose recovery). We examined $Y U C$ expression patterns in the primary root tips of plants treated with sugar (Fig. 2i-p) and compared them with the corresponding expression in plantlets grown under light for 7 days. The proYUC3-GUS plants had similar GUS signals under these three conditions (Fig. 2e, i, m). In the proYUC7-GUS plants, GUS accumulation was unaffected by whether sucrose was available in the last $24 \mathrm{~h}$ (Fig. 2f, j), but there was a considerable increase in GUS expression in response to the glucose treatment (Fig. 2n). The GUS signal decreased substantially in theproYUC8-GUS plants in the absence of sucrose (Fig. 2k), with no recovery following the glucose treatment (Fig. 2o). Although the GUS signal also decreased substantially in the proYUC9-GUS plants in the absence of sucrose (Fig. 21), the presence of glucose and sucrose resulted in a very high GUS expression level (Fig. 2p). Similar glucose effects were undetectable in the proYUC3-GUS andproYUC8-GUS plants (Fig. 2d, j-1). Assuming that sucrose or glucose in the media mimicked the effects of photoassimilates transported from shoots grown under light, we predicted that photoassimilates from shoot tissues may enhance root tip-localized auxin biosynthesis, especially by YUC7 and YUC9 in the primary root tips.

\section{Effects of photoassimilates on PIN1 and PIN2 localization in cell division zones}

In previous studies on auxin biosynthesis in roots using culture plates, sucrose was included in media from the beginning of the experiments. Thus, the sugar in media as well as photoassimilates may have stimulated auxin biosynthesis in the root tips in these earlier experiments. To test this possibility, we prepared sucrose-minus medium to ensure only the photoassimilates from shoot tissues were available to induce auxin biosynthesis. Then, we suspected that photoassimilates may be involved in PAT. We grew PIN1 GFP and PIN2-GFP plants under continuous light until 6 DAG and then transferred them to sucrose-minus medium for a 24-incubation in darkness (i.e., photoassimilate-depleted darkness treatment) to determine whether the intracellular localization of PIN1/2 and the auxin level determined using DII-VENUS were altered. The PMlocalized PIN1-GFP content was lower in response to the 24-h photoassimilate-depleted darkness treatment (Fig. 3b) than under light (Fig. 3a). This decrease was also observed in plants grown on sucrose-containing medium in darkness (Extended Data Fig. 1a, b), implying the PM-localized PIN1 content may depend on a light-dependent signal, but not sugar.

The PM-localized PIN2-GFP content also decreased following the 24-h photoassimilate-depleted darkness treatment (Fig. 3e, compared with Fig. 4d). However, in contrast to the PIN1-GFP localization results, the PM localization of PIN2-GFP was partly retained by adding sucrose to the medium in darkness (Extended Data Fig. 1c, d). These results suggested that sucrose in the growth medium or photoassimilates may lead to PM-localized PIN2.

Next, we examined DII-VENUS plants, in which fluorescence reflects the auxin concentration in an inversely proportional manner (Brunoud et al. , 2012). At 7 DAG, the DII-VENUS plants under light (autotrophic growth) had weak signals (Fig. 3g, Extended Data Fig. 2a), indicating that auxin was present in the cell division zone. In contrast, the DII-VENUS plants under light until 6 DAG and then in darkness for 1 day (photoassimilate-depleted darkness) had strong signals in the cell division zone (Fig. 3h), indicative of a low auxin concentration in the root tips. Thus, the photoassimilate supply decreased in darkness, localized auxin biosynthesis in the root tips decreased, and PAT was disrupted. This was supported by the observation that these signals were suppressed after the exogenous application of auxin or N-1-naphthylphthalamic acid (NPA) (Extended Data Fig. 2b-e).

Cell sensitivity to auxin may be determined using DR5. Earlier, we observed that DR5-GUS is produced in plants grown on $1 \%$ sucrose medium in darkness (Sakaguchi, Matsushita and Watanabe, 2019). We speculated that auxin flux in cell division zones was sustained in plants grown on sucrose-containing medium in darkness, although PIN1-mediated PAT was abnormal. It was likely that the localized auxin biosynthesis in the root tips was first activated by photoassimilates, after which the auxin induced DWF4 expression. We then examined how the subsequent BR biosynthesis in root tips leads to the maintenance of the cell division zone. 
Effects of BR on the auxin concentration in root tips and the maintenance of cell division zones

Previous studies indicated that BR signaling contributes to PAT in the primary root tips by regulating PIN localization (Li et al. , 2005; Hacham et al. , 2012; Zhang et al. , 2014; Retzeret al. , 2019). Therefore, we analyzed whether BR signaling can increase the auxin concentration in the root tips of plants grown on photoassimilate-depleted medium in darkness. The PIN1-GFP ,PIN2-GFP, and DII-VENUS plants were grown on sucrose-minus medium under continuous light until 6 DAG and then transferred to BRsupplemented medium or the mock control medium and incubated in darkness for $24 \mathrm{~h}$. The exogenous BR supply during the photoassimilate-depleted darkness treatment did not affect PIN1-GFP localization in the cell division zone (Fig. 3c), but it increased the tethering of PIN2-GFP to the PM (Fig. 3f). Additionally, DII-VENUS signals were suppressed in the stele and epidermis (Fig. 3i) and in the plants kept under continuous light (Fig. 3g), indicating the auxin supply was sufficient. This result implied that BR can bypass the signal flow disrupted by the low auxin concentration in the cell division zones, even when the photoassimilate content from the shoot or auxin biosynthesis in the root tips were insufficient.

Next, we measured the distance from the quiescent center (QC) to the border point of the cell division/elongation zone as an indicator of cell division activity (QC distance) (Silva-Navas et al. , 2016) (Extended Data Fig. 3). Compared with the light condition, the QC distance decreased during the photoassimilate-depleted darkness treatment, but not if exogenous BR was applied, suggesting BR can maintain the cell division zone size.

Considered together, these results indicated that the BR content in cell division zones contributes to the PM-localization of PIN2 and the subsequent auxin flux into the stele in seedlings under light.

\section{Brassinosteroid biosynthesis facilitated PIN2 localization in the PM of root tips and root growth}

If the interpretation above is correct, we predicted that if seedlings are treated with BR biosynthesis inhibitors under light, the PIN2 localization in the PM and the subsequent auxin flow in cell division zones would be disturbed. To test this possibility, we used BRZ220, which is a BR biosynthesis inhibitor that impedes DWF4 activity (Sekimata et al. , 2002, 2008; Nakano et al. ,2003). ThePIN2-GFP and DII-VENUS plants were grown under continuous light until 6 DAG, transferred to sucrose-minus medium with or without BRZ220, and incubated under the same light condition for $24 \mathrm{~h}$. Compared with the mock treatment, the BRZ220 treatment resulted in retarded root growth (Fig. 4a). Additionally, the PM-localized PIN2-GFP content was substantially lower following the BRZ220 treatment (Fig. 4c, f) than after the mock treatment (Fig. 4b). In response to the BRZ220 treatment, the DII-VENUS signal decreased in the epidermis, but increased slightly in the cell division zone of the stele (Fig. 4d, e, g). This result was consistent with the suppressed PAT activity following the NPA treatment (Extended Data Fig. 2d, e), confirming that BRZ220 inhibits PAT.

To demonstrate the BR-to-auxin signaling in root tips, we analyzed the PIN2-GFP localization in the dwf4102 mutant background (Fig. 4h-k). Plants grown for 6 days under continuous light were transferred to plates containing sucrose-minus agar medium with or without BR and incubated under the same light condition for $24 \mathrm{~h}$. Similar to the results of the BRZ220 treatment, PIN2-GFP was detached from the PM in the plants with the dwf4-102 background, even under light (Fig. 4j, compared with Fig. 5h); however, a 24-h treatment with exogenous BR restored the PIN2-GFP localization to the PM (Fig. 4j, k). The same BR treatment did not disrupt the PIN2 localization in the WT DWF 4 background (Fig. 4h, i). We quantified the fluorescence intensity of PIN2-GFP in the cell division zone (Fig. 4l). The fluorescence intensity decreased in the PM of the plants with the $d w f_{4}-102$ background, even under continuous light, but the exogenous $\mathrm{BR}$ treatment restored it to the WT level (Fig. 4k, l). These observations indicated that the tendency of PIN2-GFP to localize in the PM in root cell division zones depends on the BR supplied by DWF4-mediated BR biosynthesis.

We also compared the DII-VENUS localization between WT and dwf4-102 mutant plants. Because of the 
lack of PAT in the cell division zone in the dwf4-102 mutant, the DII-VENUS level increased in the stele, but decreased in the epidermis (Extended Data Fig. 4), which was in accordance with the BRZ220 treatment results (Fig. 4d, e). These findings suggested that BR in the primary root tips is indispensable for the auxin transport required for root tip growth.

\section{Discussion}

Many studies revealed the importance of auxin and BR for maintaining cell division in the root meristem (González-García et al. , 2011; Hacham et al., 2011; Chen et al. , 2014; Sakaguchi and Watanabe, 2017). We previously determined that BR biosynthesis in the root tips depends on light perception and that photoreceptor-derived uncharacterized signals from aboveground tissues induce $D W F 4$ expression in the cell elongation zone, but not in the cell division zone (Sakaguchi, Matsushita and Watanabe, 2019). Moreover, a photoassimilate-depleted darkness treatment decreases $D W F 4$ expression in the cell division zone and, unexpectedly, exogenous auxin, but not glucose, can restore DWF4 expression (Sakaguchi, Matsushita and Watanabe, 2019). Accordingly, light-dependent signals may promote DWF4 expression by increasing the auxin supply in the cell division zone. However, the source of the effective auxin content in the cell division zone was unknown.

Regarding the relationship between auxin and the root meristem, it was reported that auxin biosynthesis in the root tip, but not in the aboveground tissue, is indispensable for the root meristem (Chenet al. , 2014). Moreover, glucose treatments can increase the auxin level by promoting the expression of genes encoding auxin biosynthesis-related enzymes (Sairanen et al. , 2013). On the basis of our previous research, we investigated the relationship between photoassimilate translocation (i.e., shoot-to-root signaling) and YUCs in promoting root growth. A 3-h glucose treatment after a 24-h photoassimilate-depleted darkness treatment revealed that sugar can replace photoassimilates as inducers of $Y U C 7 / 9$ expression in the root tips. Consistent with this finding, we observed that $D W F 4$ expression in the root tips decreased in the yuc $Q$ mutant background. This suggested that after photoassimilates from the aboveground tissues reach the root tips, auxin biosynthesis is induced, with the resulting auxin activating DWF4 expression. In accordance with this possibility, a treatment with Kyn, which is an auxin biosynthesis inhibitor, suppressed DWF4 accumulation in the cell division zone and inhibited root growth.

Interestingly, recent studies elucidated the relationship between BR and auxin in the root tissue. The nitrogen deficiency induces BR-dependent auxin biosynthesis in the lateral root tips and the subsequent elongation (Jia, Giehl and von Wirén, 2021). BR in the root tip promotes auxin biosynthesis and suppresses auxin signaling, thereby promoting root meristem activity (Ackerman-Lavert et al. , 2021). Integrating these earlier findings with our results, we propose that the biosynthesis of auxin and BR in the cell division zone may establish a positive regulatory feedback loop initiated by photoassimilates.

We have been studying how light-induced localized BR biosynthesis influences root growth. Earlier, it was reported that BR signaling inhibits PIN2 endocytosis (Retzer et al. , 2019). Furthermore, PIN2 revealed to have a crucial role in the auxin transport through the epidermis that maintains the root growth rate (Hu et al. , 2021). Other studies indicated that darkness promotes the detachment of PIN1/2 from the PM (Laxmi et al. , 2008; Sassi et al. , 2012; Zhanget al. , 2014). On the basis of these reports, we assumed that the lightinduced BR supply promotes PIN2 localization in the PM and the subsequent root growth. We observed that the photoassimilate-depleted darkness treatment was not conducive to PAT, but exogenous BR can recover the PAT in the epidermis of cell division zones. Moreover, the BRZ220 treatment of PIN2-GFP and DII-VENUS plants (dwf-102 background) revealed that BR is required for PAT and the subsequent auxin level in the stele. In particular, BR biosynthesis and signaling in the cell division zone are incorporated into the light-induced auxin signaling pathway. It was reported that PIN2 facilitates auxin flux into the stele (Hu et al. , 2021). Therefore, it is likely that the PAT potentiated by BR-dependent PIN2 is critical for the auxin flux into the stele.

In this study, we produced evidence that the interplay between auxin and BR in the root tip cell division zone is induced by light-dependent signals from the shoot tissue. Integrating our results with those of recent 
investigations, we propose a model (Fig. 5) in which light-signaling relay systems in cell division and elongation zones promote BR biosynthesis. Recent study revealed that the BR levels optimal for root growth differ between the cell division and cell elongation zones (Vukašinović et al. , 2021). During the cell stage transitions in growing roots, the role of BR signaling and the required BR level may vary among locations. Considering the difference in the required BR content between the cell division and cell elongation zones, it is likely that BR biosynthesis is differentially regulated in these two regions, which may explain the diversity in the localized BR concentration in the root tips and the subsequent auxin transport (Hacham et al. , 2012; Retzer et al. , 2019; Vukašinovićet al. , 2021).

The description of light-dependent root growth would help comprehension of the determinate growth for the whole root system in nature. As the primary root elongates and the lateral roots increase, the lightdependent signaling would distribute in each branch. It means that the signaling from the shoot tissue for the root growth decreased according to the distance and might dominate the whole root architecture.

\section{Acknowledgments}

We thank Hiroyuki Kasahara and Yunde Zhao for providing theproYUC-GUS and yucQ stocks. We thank Michitaka Notaguchi for the lecture on micrografting technique and the supply of micrografting chips. We thank Edanz (https://jp.edanz.com/ac) for editing a draft of this manuscript.

\section{Author contributions}

J.S. and Y.W. conceived and designed the experiments. J.S. conducted the experiments and analyzed the results. J.S. took all the photographs. J.S. and Y.W. wrote the manuscript.

\section{Competing financial interests}

The authors declare no competing financial interests.

\section{Methods}

\section{Plant materials and growth conditions}

In addition to Arabidopsis Col-0 (WT control), we used the following Arabidopsis marker lines and mutant lines in this study: DWF4-GUS plants (Sakaguchi and Watanabe, 2017),PIN1-GFP plants(Benková et al. , 2003), PIN2-GFPplants (Xu and Scheres, 2005), DII-VENUS plants(Brunoud et al. , 2012), pro YUC-GUS transgenic lines (Chen et al. , 2014), homozygotes of the cry1-304phyB-9 double mutant (Shikataet al. , 2014), and homozygotes of the yuc3yuc5yuc 7 yuc8yuc9quintuple mutant (Chen et al. , 2014). We incorporatedPIN2GFP or DII-VENUS into dwf4-102 mutants to analyze PIN2 localization and auxin levels in the cell division zone in the BR-deficient background. Only two plants from $249 d w f 4$ segregants in the $\mathrm{F}_{2}$ population crossed withdwf4-102 and DII-VENUS plants produced detectable VENUS fluorescence.

Seeds were surface-sterilized with $70 \%(\mathrm{v} / \mathrm{v})$ ethanol for $3 \mathrm{~min}$, rinsed four times with distilled water, and incubated in $0.05 \%(\mathrm{v} / \mathrm{v})$ plant preservative mixture (Plant Cell Technology, Washington, DC, USA; http://www.plantcelltechnology.com/) for $24 \mathrm{~h}$ at room temperature in darkness. Sterilized seeds were germinated in plates containing half-strength MS basal salt medium, $\mathrm{B}_{5}$ vitamins, $2 \mathrm{mM} \mathrm{2-}$ morpholinoethanesulfonic acid monohydrate $(\mathrm{pH} 5.8)$, and $1.5 \%(\mathrm{w} / \mathrm{v})$ agarose. The plates were positioned vertically and incubated at $22{ }^{\circ} \mathrm{C}$ under continuous white light (FHF32EX-N-H, a three-wavelength emission-type fluorescent lamp, with a front incident strength of $25 \mu \mathrm{mol} \mathrm{m}^{-2} \mathrm{~s}^{-1}$; Panasonic, Osaka, Japan; http://panasonic.jp/light).

\section{Micrografting}

Seed sterilization was described above. Each sterilized seed was settled in a pocket of upper position on the micro-grafting chip with $0.08 \%$ agar (Star agar L-grade 01, Rikaken, https://www.rikaken.co.jp/). A series of micrografting processes followed technical guidance (Tsutsuiet al. , 2020). The adventitious roots generated from the scion side were removed at 6 days after grafting, then the grafted plantlets were vertically settled in MS agar plates. 


\section{Sugar recovery test and photoassimilate-depleted darkness treatment}

For the sugar recovery test, plants were grown under darkness until 6 DAG in vertical plates containing agar with $1 \%(\mathrm{w} / \mathrm{v})$ sucrose. The samples were rinsed five times with distilled water and transferred to sucrose-minus liquid medium for a 24-h incubation in darkness. The glucose-containing liquid medium treatment was performed as previously described (Gupta, Singh and Laxmi, 2015). For the photoassimilatedepleted darkness treatment, plants were grown in vertical plates containing sucrose-minus agar medium under continuous light until 6 DAG. The samples were transferred to plates containing fresh sucrose-minus agar medium and then incubated in a vertical position for $24 \mathrm{~h}$ in darkness for the consumption of endogenous photoassimilates (photoassimilate-depleted darkness treatment).

\section{Phytohormone or biosynthesis inhibitor treatment}

Plants were grown for up to 6 DAG in vertical plates and then transferred to plates with MS agar medium without sucrose, but with other chemicals, for a 24-h incubation under light or in darkness for the consumption of photoassimilates. The working concentrations of the chemicals used in this study were as follows: L-kynurenine (Kyn: 10, 50, and $100 \mu \mathrm{M}), 2$,4-dichlorophenoxyacetic acid (2,4-D: $1 \mu \mathrm{M})$, 1-naphthaleneacetic acid (NAA: $1 \mu \mathrm{M}$ ), N-1-naphthylphthalamic acid (NPA: 10 and $100 \mu \mathrm{M}$ ), brassinolide (BL: $0.4 \mathrm{nM}$ ), and brassinazole 220 (BRZ220: $5 \mu \mathrm{M}$ ). The same volume of the blank solvent (DMSO or ethanol) was used for the mock treatment. The plants in plates containing medium supplemented with BRZ220 had bent roots that were detached from the agar medium. Therefore, a transparent polyethylene film was used to keep the root tips in the agar medium.

\section{Histochemical staining of GUS plants}

To analyze GUS staining patterns, plant samples were fixed in $70 \%$ acetone for 15 min on ice and then incubated in $100 \mathrm{mM}$ sodium phosphate buffer (pH 7.0) containing $10 \mathrm{mM}$ sodium EDTA, $5 \mathrm{mM}$ potassium ferrocyanide, $5 \mathrm{mM}$ potassium ferricyanide, $0.1 \%$ (v/v) Triton X-100, and $0.5 \mathrm{mg} \mathrm{ml}^{-1} 5$-bromo-4chloro-3-indolyl-b-D-glucuronic acid at $37{ }^{\circ} \mathrm{C}$ overnight, depending on the staining intensity as previously described(Sakaguchi and Watanabe, 2017).

\section{Microscopy}

A BX60 microscope (Olympus; http://www.olympus-global.com/en/) equipped with a DFC480 CCD digital camera (Leica Microsystems, Wetzlar, Germany; https://www.leica-microsystems.com/) was used to analyze GUS staining. A C2 plus confocal microscope (Nikon, Tokyo, Japan; http://www.nikon.com/index.htm) was used to examine root tips stained with propidium iodide (PI) and to examine fluorescent markers in plants. The roots of plants at 7 DAG were stained in $2.5 \mu \mathrm{g} \mathrm{ml} \mathrm{m}^{-1} \mathrm{PI}$ for 5-6 min and then examined after an excitation step involving the Sapphire 561-nm laser. The PI signal was detected using a 575-615-nm bandpass filter. The GFP and DII-VENUS fluorescence after the excitation by the Sapphire 488-nm laser was detected using a 500-550-nm bandpass filter.

\section{qRT-PCR analysis}

Root tips (approximately $5 \mathrm{~mm}$ long) were collected from plants at 7 DAG for the qRT-PCR analysis. Total RNA was extracted using the NucleoSpin@ RNA kit (MACHEREY-NAGEL; http://www.mnnet.com/). First-strand cDNA was synthesized using the PrimeScript RT reagent Kit with gDNA Eraser (Perfect Real Time) (TAKARA-BIO; http://www.takara-bio.co.jp/). The KAPA SYBR(r) FAST qPCR Kit (KAPABIOSYSTEMS; https://www.kapabiosystems.com/) was used for qRT-PCR analyses, which were performed in duplicate on the QuantStudio 3 Real Time PCR System (Applied Biosystems; http://www.thermofisher.com/). The 10- $\mu$ l qRT-PCR mixture included 1- $\mu$ l first-strand cDNA (from 20 ng total RNA) as the template. Data were analyzed using the AppConnect online service (ThermoFisher Scientific, Waltham, MA, USA; www.thermofisher.com/).

\section{Root apical meristem activity analysis}


Cell division in the primary root tips was assessed according to the QC distance (González-García et al. , 2011). In this study, we marked the border between the meristem and elongating cells, where the adjacent cells were more than $50 \%$ longer, and measured the distance from the QC to the border position.

\section{Measurement of fluorescence intensity}

The fluorescence intensity of PIN2-GFP and DII-VENUS in the cell division zone was measured using the ImageJ software (https://imagej.nih.gov/ij/).

\section{Statistical analysis}

All statistical analyses were completed using Microsoft Excel 2016. Significant differences between two independent samples were determined according to Student's $t$-test. Significant differences in multiple comparisons were determined using the Tukey-Kramer method.

\section{References}

Ackerman-Lavert, M. et al. (2021) 'Auxin requirements for a meristematic state in roots depend on a dual brassinosteroid function', Current Biology , pp. 1-11. doi: 10.1016/j.cub.2021.07.075.

Azpiroz, R. et al. (1998) 'An Arabidopsis Brassinosteroid-Dependent Mutant Is Blocked in Cell Elongation', The Plant Cell , 10(2), pp. 219-230. doi: 10.1105/tpc.10.2.219.

Benková, E. et al. (2003) 'Local, Efflux-Dependent Auxin Gradients as a Common Module for Plant Organ Formation', Cell , 115(5), pp. 591-602. doi: 10.1016/S0092-8674(03)00924-3.

Brunoud, G. et al. (2012) 'A novel sensor to map auxin response and distribution at high spatio-temporal resolution', Nature , 482(7383), pp. 103-106. doi: 10.1038/nature10791.

Chen, Q. et al. (2014) 'Auxin Overproduction in Shoots Cannot Rescue Auxin Deficiencies in Arabidopsis Roots', Plant and Cell Physiology, 55(6), pp. 1072-1079. doi: 10.1093/pcp/pcu039.

Cheng, Y., Dai, X. and Zhao, Y. (2006) 'Auxin biosynthesis by the YUCCA flavin monooxygenases controls the formation of floral organs and vascular tissues in Arabidopsis', Genes 63 Development, 20(13), pp. 1790-1799. doi: 10.1101/gad.1415106.

Choe, S. et al. (1998) 'The DWF4 Gene of Arabidopsis Encodes a Cytochrome P450 That Mediates Multiple 22 $\alpha$-Hydroxylation Steps in Brassinosteroid Biosynthesis', The Plant Cell , 10(2), pp. 231-243. doi: 10.1105/tpc.10.2.231.

Fujioka, S., Takatsuto, S. and Yoshida, S. (2002) 'An Early C-22 Oxidation Branch in the Brassinosteroid Biosynthetic Pathway',Plant Physiology, 130(2), pp. 930-939. doi: 10.1104/pp.008722.

Fujita, S. et al. (2006) 'Arabidopsis CYP90B1 catalyses the early C-22 hydroxylation of C 27 , C 28 and C 29 sterols', The Plant Journal , 45(5), pp. 765-774. doi: 10.1111/j.1365-313X.2005.02639.x.

González-García, M.-P. et al. (2011) 'Brassinosteroids control meristem size by promoting cell cycle progression in Arabidopsis roots',Development, 138(5), pp. 849-859. doi: 10.1242/dev.057331.

Gupta, A., Singh, M. and Laxmi, A. (2015) 'Multiple Interactions between Glucose and Brassinosteroid Signal Transduction Pathways in Arabidopsis Are Uncovered by Whole-Genome Transcriptional Profiling', Plant Physiology , 168(3), pp. 1091-1105. doi: 10.1104/pp.15.00495.

Ha, J.-H. et al. (2018) 'Shoot phytochrome B modulates reactive oxygen species homeostasis in roots via abscisic acid signaling in Arabidopsis', The Plant Journal , 94(5), pp. 790-798. doi: 10.1111/tpj.13902.

Hacham, Y. et al. (2011) 'Brassinosteroid perception in the epidermis controls root meristem size', Development , 138(5), pp. 839-848. doi: 10.1242/dev.061804.

Hacham, Y. et al. (2012) 'BRI1 activity in the root meristem involves post-transcriptional regulation of PIN auxin efflux carriers',Plant Signaling and Behavior , 7(1). doi: 10.4161/psb.7.1.18657. 
He, W. et al. (2011) 'A small-molecule screen identifies L-kynurenine as a competitive inhibitor of TAA1/TAR activity in ethylene-directed auxin biosynthesis and root growth in Arabidopsis.', The Plant Cell , 23(11), pp. 3944-60. doi: 10.1105/tpc.111.089029.

$\mathrm{Hu}$, Yangjie et al. (2021) 'Cell kinetics of auxin transport and activity in Arabidopsis root growth and skewing', Nature Communications , 12(1), pp. 1-13. doi: 10.1038/s41467-021-21802-3.

Jia, Z., Giehl, R. F. H. and von Wirén, N. (2021) 'Local auxin biosynthesis acts downstream of brassinosteroids to trigger root foraging for nitrogen', Nature Communications , 12(1), p. 5437. doi: 10.1038/s41467021-25250-x.

Kim, G.-T. et al. (2005) 'CYP90C1 and CYP90D1 are involved in different steps in the brassinosteroid biosynthesis pathway in Arabidopsis thaliana', The Plant Journal , 41(5), pp. 710-721. doi: 10.1111/j.1365313X.2004.02330.x.

Kircher, S. and Schopfer, P. (2012) 'Photosynthetic sucrose acts as cotyledon-derived long-distance signal to control root growth during early seedling development in Arabidopsis', Proceedings of the National Academy of Sciences , 109(28), pp. 11217-11221. doi: 10.1073/pnas.1203746109.

Laxmi, A. et al. (2008) 'Light Plays an Essential Role in Intracellular Distribution of Auxin Efflux Carrier PIN2 in Arabidopsis thaliana', PLoS ONE . Edited by F. Berger, 3(1), p. e1510. doi: 10.1371/journal.pone.0001510.

Li, J. et al. (2001) 'BIN2, a New Brassinosteroid-Insensitive Locus in Arabidopsis', Plant Physiology , 127(1), pp. 14-22. doi: 10.1104/pp.127.1.14.

Li, J. and Chory, J. (1997) 'A putative leucine-rich repeat receptor kinase involved in brassinosteroid signal transduction', Cell , 90(5), pp. 929-938. doi: 10.1016/s0092-8674(00)80357-8.

Li, L. et al. (2005) 'Brassinosteroids stimulate plant tropisms through modulation of polar auxin transport in Brassica and Arabidopsis', Plant Cell , 17(10), pp. 2738-2753. doi: 10.1105/tpc.105.034397.

Maharjan, P. M. et al. (2014) 'Arabidopsis gulliver1/superroot2-7 identifies a metabolic basis for auxin and brassinosteroid synergy', The Plant Journal , 80(5), pp. 797-808. doi: 10.1111/tpj.12678.

Mashiguchi, K. et al. (2011) 'The main auxin biosynthesis pathway in Arabidopsis', Proceedings of the National Academy of Sciences , 108(45), pp. 18512-18517. doi: 10.1073/pnas.1108434108.

Matosevich, R. et al. (2020) 'Local auxin biosynthesis is required for root regeneration after wounding', Nature Plants , 6(8), pp. 1020-1030. doi: 10.1038/s41477-020-0737-9.

Moore, B. (2003) 'Role of the Arabidopsis Glucose Sensor HXK1 in Nutrient, Light, and Hormonal Signaling', Science, 300(5617), pp. 332-336. doi: 10.1126/science.1080585.

Nakano, T. et al. (2003) 'The Influence of Chemical Genetics on Plant Science: Shedding Light on Functions and Mechanism of Action of Brassinosteroids Using Biosynthesis Inhibitors', Journal of Plant Growth Regulation , 22(4), pp. 336-349. doi: 10.1007/s00344-003-0065-0.

Noguchi, T. et al. (2000) 'Biosynthetic Pathways of Brassinolide in Arabidopsis', Plant Physiology, 124(1), pp. 201-210. doi: 10.1104/pp.124.1.201.

Ohnishi, T. et al. (2006) 'C-23 Hydroxylation by Arabidopsis CYP90C1 and CYP90D1 Reveals a Novel Shortcut in Brassinosteroid Biosynthesis', The Plant Cell , 18(11), pp. 3275-3288. doi: 10.1105/tpc.106.045443.

Retzer, K. et al. (2019) 'Brassinosteroid signaling delimits root gravitropism via sorting of the Arabidopsis PIN2 auxin transporter',Nature Communications , 10(1), p. 5516. doi: 10.1038/s41467-019-13543-1.

Rolland, F., Moore, B. and Sheen, J. (2002) 'Sugar Sensing and Signaling in Plants', The Plant Cell , 14(suppl 1), pp. S185-S205. doi: 10.1105/tpc.010455. 
Sairanen, I. et al. (2013) 'Soluble Carbohydrates Regulate Auxin Biosynthesis via PIF Proteins in Arabidopsis', The Plant Cell , 24(12), pp. 4907-4916. doi: 10.1105/tpc.112.104794.

Sakaguchi, J., Matsushita, T. and Watanabe, Y. (2019) 'DWARF4 accumulation in root tips is enhanced via blue light perception by cryptochromes', Plant, Cell \& Environment , 42(5), pp. 1615-1629. doi: 10.1111/pce.13510.

Sakaguchi, J. and Watanabe, Y. (2017) 'Light perception in aerial tissues enhances DWF4 accumulation in root tips and induces root growth', Scientific Reports , 7(1), p. 1808. doi: 10.1038/s41598-017-01872-4.

Sassi, M. et al. (2012) 'COP1 mediates the coordination of root and shoot growth by light through modulation of PIN1- and PIN2-dependent auxin transport in Arabidopsis', Development, 139(18), pp. 3402-3412. doi: $10.1242 /$ dev.078212.

Sekimata, K. et al. (2002) 'Brz220 a novel brassinosteroid biosynthesis inhibitor: stereochemical structureactivity relationship', Tetrahedron: Asymmetry, 13(17), pp. 1875-1878. doi: 10.1016/S0957-4166(02)004755 .

Sekimata, K. et al. (2008) 'Brz220 Interacts with DWF4, a Cytochrome P450 Monooxygenase in Brassinosteroid Biosynthesis, and Exerts Biological Activity', Bioscience, Biotechnology, and Biochemistry , 72(1), pp. 7-12. doi: 10.1271/bbb.70141.

Shikata, H. et al. (2014) 'Phytochrome controls alternative splicing to mediate light responses in Arabidopsis', Proceedings of the National Academy of Sciences , 111(52), pp. 18781-18786. doi: 10.1073/pnas.1407147112.

Shimada, Y. et al. (2003) 'Organ-Specific Expression of Brassinosteroid-Biosynthetic Genes and Distribution of Endogenous Brassinosteroids in Arabidopsis', Plant Physiology , 131(1), pp. 287-297. doi: 10.1104/pp.013029.

Silva-Navas, J. et al. (2016) 'Flavonols Mediate Root Phototropism and Growth through Regulation of Proliferation-to-Differentiation Transition', The Plant Cell , 28(6), pp. 1372-1387. doi: 10.1105/tpc.15.00857.

Stepanova, A. N. et al. (2008) 'TAA1-Mediated Auxin Biosynthesis Is Essential for Hormone Crosstalk and Plant Development', Cell , 133(1), pp. 177-191. doi: 10.1016/j.cell.2008.01.047.

Sugawara, S. et al. (2009) 'Biochemical analyses of indole-3-acetaldoxime-dependent auxin biosynthesis in Arabidopsis', Proceedings of the National Academy of Sciences , 106(13), pp. 5430-5435. doi: $10.1073 /$ pnas.0811226106.

Sun, L. et al. (2020) 'PIN-LIKES Coordinate Brassinosteroid Signaling with Nuclear Auxin Input in Arabidopsis thaliana',Current Biology , 30(9), pp. 1579-1588.e6. doi: 10.1016/j.cub.2020.02.002.

Tian, H. et al. (2018) 'Auxin-BR interaction regulates plant growth and development', Frontiers in Plant Science , 8(January), pp. 1-8. doi: 10.3389/fpls.2017.02256.

Tsutsui, H. et al. (2020) 'Micrografting device for testing systemic signaling in Arabidopsis', The Plant Journal , 103(2), pp. 918-929. doi: 10.1111/tpj.14768.

Vukašinović, N. et al. (2021) 'Local brassinosteroid biosynthesis enables optimal root growth', Nature Plants , 7(5), pp. 619-632. doi: 10.1038/s41477-021-00917-x.

$\mathrm{Xu}$, J. and Scheres, B. (2005) 'Dissection of Arabidopsis ADP-RIBOSYLATION FACTOR 1 Function in Epidermal Cell Polarity', The Plant Cell , 17(2), pp. 525-536. doi: 10.1105/tpc.104.028449.

Yamada, M. et al. (2009) 'The TRANSPORT INHIBITOR RESPONSE2 Gene Is Required for Auxin Synthesis and Diverse Aspects of Plant Development', Plant Physiology , 151(1), pp. 168-179. doi: 10.1104/pp.109.138859. 
Zhang, K. X. et al. (2014) 'Proper PIN1 distribution is needed for root negative phototropism in Arabidopsis', PLoS ONE , 9(1). doi: 10.1371/journal.pone.0085720.

Zhao, Y. et al. (2001) 'A role for flavin monooxygenase-like enzymes in auxin biosynthesis.', Science , 291(5502), pp. 306-9. doi: 10.1126/science.291.5502.306.

Figure legends

Fig. 1 | Micrografting with the cry1phyB scions has less impact on the auxin and BR in the cell division zone in the primary root tip.

(a ) Image of grafted seedlings at 13 days after grafting. Compared to the WT scions, cry1phyB scions indicated the retarded root growth. Arrowheads indicated the graft junctions. (b-c ) DWF4-GUS accumulation was detected in the cell division zone but not in the cell elongation zone with the cry1phyB scions. (d-e ) PIN1-GFP localization in the PM was observed in the cell division zone with the WT scions and cry1phyB scions. Scale bars represent $10 \mathrm{~mm}(\mathbf{a}), 50 \mu \mathrm{m}$ (b-e ).

Fig. 2 DWF4 accumulation in the cell division zone depended on the light-induced auxin supply.

(a-d ) The 24-h kynurenine (Kyn) treatment under light decreased DWF4 accumulation in the cell division zone. (e ) The 24-h darkness treatment restricted DWF4 accumulation near the apex. (f ) The 24-h and 48-h Kyn treatment under light retarded root growth $[\mathrm{n}=26]$. (g ) The qRT-PCR analysis revealed that $D W F 4$ expression in the root tip decreased in the yucQmutant. Error bars represent standard deviations. Means denoted by the same letter are not significantly different $(\mathrm{P}<0.05)$ according to the Tukey-Kramer method. ${ }^{*} \mathrm{P}<0.05$. Arrowheads indicate the first cell of the elongation zone. Scale bars represent $50 \mu \mathrm{m}$.

Fig. 3 | YUC3, 7, and 9 promoter activities in the primary root tips of plants grown on sucrose-minus medium in darkness.

(a-d) At 7 DAG, proYUC-GUS levels in plants grown on $1 \%$ sucrose medium (Suc. 1\%) under continuous light. Except forproYUC8, YUC expression was detected in the root tip. (e -h ) At 7 DAG, proYUCGUS levels in plants grown on Suc. $1 \%$ medium in continuous darkness. YUC expression was detected in the root tip. (i -1 ) The 24-h no-sucrose/glucose treatment. Compared with the plants grown in the presence of sucrose, YUC8 and YUC9 expression levels decreased in the primary root tips of plants grown on the sucrose-minus medium. (m-p ) The 3-h glucose recovery treatment after a 24-h no-sucrose/glucose treatment. Both $\mathrm{YUC}^{r}$ and $Y U C 9$ were highly expressed following the glucose treatment. Arrowheads indicate the first cell of the elongation zone. Scale bars represent $100 \mu \mathrm{m}$.

Fig. 4 The lack of photoassimilates affected the PAT and auxin level in the cell division zone, but the exogenous BR treatment diminished these effects.

(a -c ) PIN1-GFP, (d -f )PIN2-GFP, and (g -i ) DII-VENUS plants were grown on sucrose-minus medium under continuous light until 6 DAG to attain autotrophic growth. Left panels: plants were placed under light for another $24 \mathrm{~h}(+24 \mathrm{~h}$ light; autotroph/plants a , d , and $\mathbf{g})$. Middle panels: plants were placed in darkness for $24 \mathrm{~h}(+24 \mathrm{~h}$ dark; sucrose-minus/plants b , e, andh $)$. Right panels: plants on BR-supplemented medium were placed in darkness for $24 \mathrm{~h}(+24 \mathrm{~h}$ dark; sucrose-minus with BR supplied/plantsc, f , and i ). PIN1 and PIN2 localization was disrupted and DII-VENUS degradation was suppressed by a lack of sucrose in the cell division zones of plants grown on sucrose-minus medium in darkness. The application of exogenous BR in darkness restored the localization of PIN2, but not PIN1, to the PM, while also suppressing the darkness-induced DII-VENUS signal in the cell division zone. Arrowheads indicate the first cell of the elongation zone. Scale bars represent $50 \mu \mathrm{m}$.

Fig. 5 | PIN2 localization in the PM depended on the BR supply in the cell division zone.

(a ) The 24-h BRZ220 treatment under light retarded root growth $[\mathrm{n}=20]$. (b -c ) The 24-h BRZ220 treatment under light decreased the PIN2 localization in the PM in the cell division zone. (d -e ) The 24-h 
BRZ220 treatment under light did not significantly affect DII-VENUS like the effect of the photoassimilatedepleted darkness treatment. (f ) The BRZ220 treatment decreased the PIN2 localization in the PM [n $=50]$. (g ) The BRZ220 treatment decreased the DII-VENUS signal in the nuclei in the epidermis, but increased the signal in the stele $[\mathrm{n}=50]$. (h -i ) PIN2-GFP localized in the PM in the cell division zone in response to the mock treatment, and the 24-h exogenous BR treatment did not suppress the PIN2 localization. ( $\mathbf{j}-\mathbf{k}$ ) PIN2-GFP localization was severely affected in the dwf4-102 mutant background in response to the mock treatment, but it was restored by the 24-h exogenous BR treatment. 1, PIN2 fluorescence intensity in the PM decreased in the dwf4-102 mutant background, but it was restored by the 24-h exogenous BR treatment $[\mathrm{n}=50]$. Error bars represent standard deviations. ${ }^{*} \mathrm{P}<0.001$. Scale bars represent $50 \mu \mathrm{m}$.

Fig. 6 | Model of the auxin-BR interaction in the root tip initiated by light-dependent signaling.

The shoot tissue produces two different signals perceived by the primary root tip. In the cell elongation zone, the photoreceptor-derived signal promotes DWF4 accumulation and the subsequent BR biosynthesis. In the cell division zone, photoassimilates promote the expression of several $Y U C$ genes. Additionally, auxin synthesized in the root tip promotes DWF 4 expression and the subsequent BR biosynthesis. Next, BR induces the PIN2 localization in the PM. Moreover, BR contributes to the auxin flux into the stele because it establishes the PAT into the cell division zone. It is likely that the PAT via PIN2 enhanced by BR signaling mediates this auxin flux.

\section{Extended Data Figure Legends}

Extended Data Fig. 1 | Decreased PIN1/2 localization in the primary root tip in darkness.

PIN1 localization in the primary root tip under light (a ) and in darkness (b ). Decreased PIN1 localization in the stele PM in darkness. PIN2 localization in the primary root tip under light (c) and in darkness (d ). Decreased PIN2 localization in the epidermis/cortex PM in darkness. Arrowheads indicate the first cell of the elongation zone. Scale bars represent $50 \mu \mathrm{m}$.

Extended Data Fig. 2| DII-VENUS signals in the primary root tip following the auxin-related treatment.

(a ) The DII-VENUS signal was stronger in the epidermal cells than in the cortex or the stele. (b ) The DIIVENUS signal in the epidermal cells, but not in the stele, decreased substantially after the 2,4-D treatment. (c) The DII-VENUS signal was undetectable in the primary root tip following the NAA treatment. (d -e ) The DII-VENUS signal in the epidermal cells, but not in the stele, decreased in response to NPA in a dose-dependent manner. (f ) Intensity of the DII-VENUS fluorescence in the epidermis and stele [n $=50]$. Arrowheads indicate the first cell of the elongation zone. Scale bars represent $50 \mu \mathrm{m}$.

Extended Data Fig. 3 | The QC distance decreased after the 24-h darkness treatment, but was restored by the exogenous BR treatment.

The QC distance was measured as the index of the meristematic region size in the root tip [n $=24]$. The photoassimilate-depleted darkness treatment decreased the $\mathrm{QC}$ distance, but the exogenous $\mathrm{BR}$ treatment restored the QC distance.

Extended Data Fig. $4 \mid$ DII-VENUS fluorescence in the cell division zone increased in the dwf4-102 mutants.

Compared with the WT plants, the DII-VENUS signal in the stele increased significantly in the dwf4-102 mutants under light, similar to the effects of the photoassimilate-depleted darkness treatment. Scale bars represent $50 \mu \mathrm{m}$. 
Fig. 1

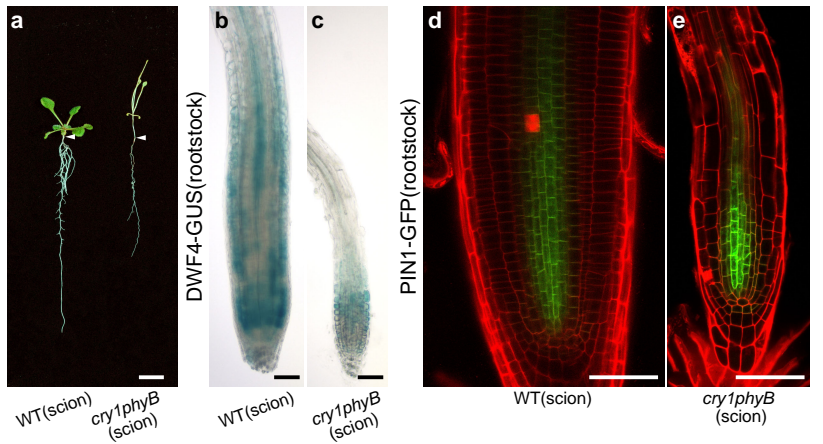

Fig. 1 | Micrografting with the cry1phyB scions has less impact on the auxin and BR in the cell division zone in the primary root tip.

(a) Image of grafted seedlings at 13 days after grafting. Compared to the WT scions, cry1phyB scions indicated the retarded root growth. Arrowheads indicated the graft junctions. (b-c) DWF4-GUS accumulation was detected in the cell division zone but not in the cell elongation zone with the cry1phyB scions. (d-e) PIN1-GFP localization in the PM was observed in the cell division zone with the WT scions and crylphyB scions.

Scale bars represent $10 \mathrm{~mm}$ (a), $50 \mu \mathrm{m}$ (b-e). 
Fig. 2
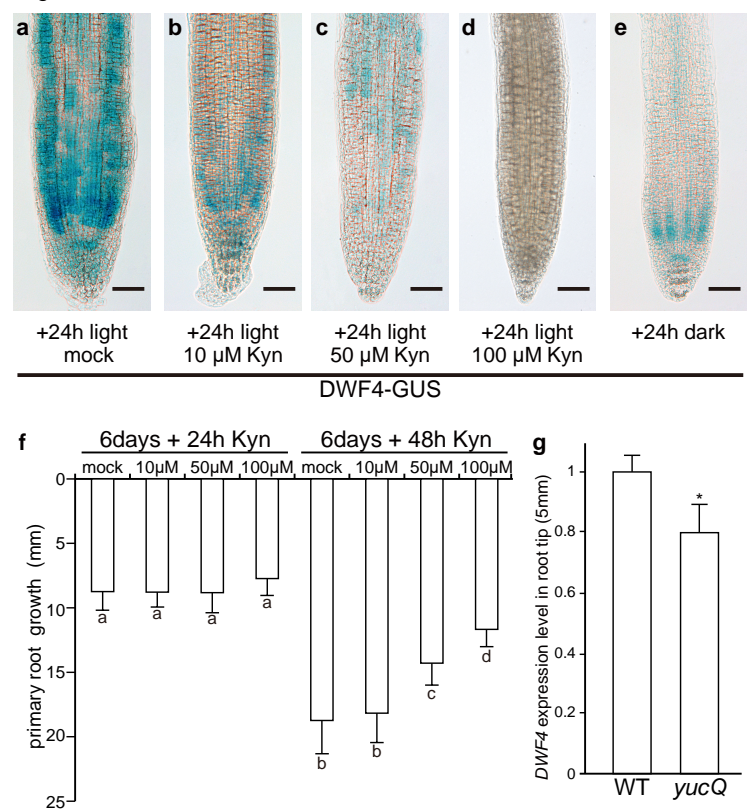

Fig. 2 | DWF4 accumulation in the cell division zone depended on the light induced auxin supply. (a-d) The 24-h of kynurenine (Kyn) treatment under light decreased DWF4 accumulation in the cell division zone. (e) The 24-h darkness treatment restricted DWF4 accumulation near the apex. (f) The 24-h and 48-h Kyn treatment under light retarded root growth $(\mathrm{n}=26)$. (g) The qRT-PCR analysis revealed that DWF4 expression in the root tip decreased in the yucQ mutant. Error bars represent standard deviations. ${ }^{*} \mathrm{P}<0.05$. Arrowheads indicate the first cell of the elongation zone. Scale bars represent $50 \mu \mathrm{m}$ 
Fig. 3
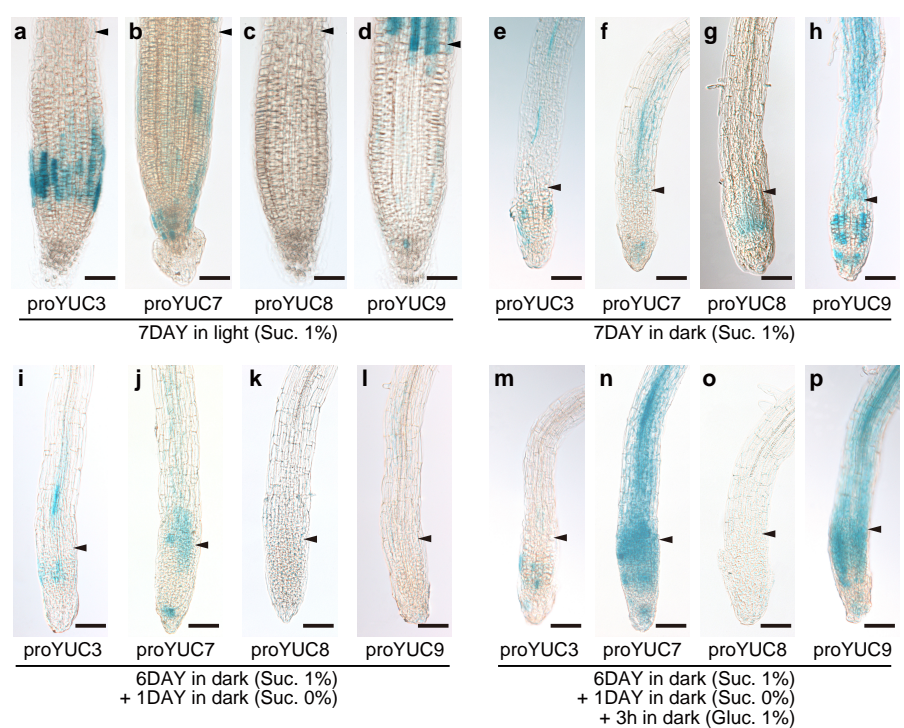

Fig. 3 | YUC3, 7, and 9 promoter activities in the primary root tips of plants grown on sucrose-minus medium in darkness.

(a-d) At 7 DAG, proYUC-GUS levels in plants grown on 1\% sucrose medium (Suc. 1\%) under continuous light. Except for proYUC8, YUC expression was detected in the root tip. (e-h) At 7 DAG, proYUC-GUS levels in plants grown on Suc. $1 \%$ medium in continuous darkness. YUC expression was detected in the root tipd. (i-l) The 24-h no-sucrose/glucose treatment. Compared with the plants grown in the presence of sucrose, YUC8 and YUC9 expression levels decreased in the primary root tips of plants grrown on the sucrose-minus medium. (m-p) The 3-h glucose recovery treatment after a 24-h no-sucrose/glucose treatment. Both YUC7 and YUC9 were highly expressed following the glucose treatment.

Arrowheads indicate the first cell of the elongation zone. Scale bars represent $100 \mu \mathrm{m}$. 

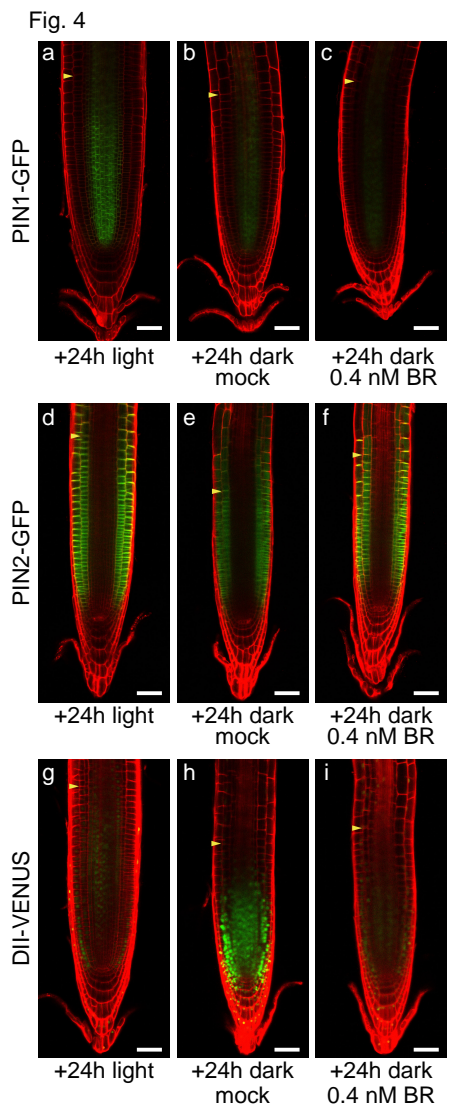

Fig. 4 | The lack of photoassimilates affected the PAT and auxin level in the cell division zone, but the exogenous BR treatment diminished these effects.

(a-c) PIN1-GFP, (d-f) PIN2-GFP, and (g-i) DII-VENUS plants were grown on sucrose-minus medium under continuous

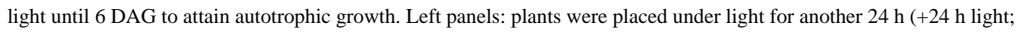
autotroph/plants a, d, and g). Middle panels: plants were placed in darkness for $24 \mathrm{~h}$ (+24 h dark; sucrose-minus/plants b, e, and h). Right panels: plants on BR-supplemented medium were placed in darkness for $24 \mathrm{~h}$ (+24h dark; sucrose-minus with BR supplied/plants c, f, and i). PIN1 and PIN2 localization was disrupted and DII-VENUS degradation was suppressed by sucrose-minus medium in darkness. The application of exogenous BR in darkness restored the localization of PIN2, but not PIN1, to the PM, while also suppressing the darkness-induced DII-VENUS signal in the cell division zone.

Arrowheads indicate the first cell of the elongation zone. Scale bars represent $50 \mu \mathrm{m}$

\section{Hosted file}

Figure5.pdf available at https://authorea.com/users/459494/articles/555748-cell-divisionzones-are-maintained-by-the-photoassimilate-auxin-brassinosteroid-polar-auxin-transportsignal-relay-in-arabidopsis-root-tips 


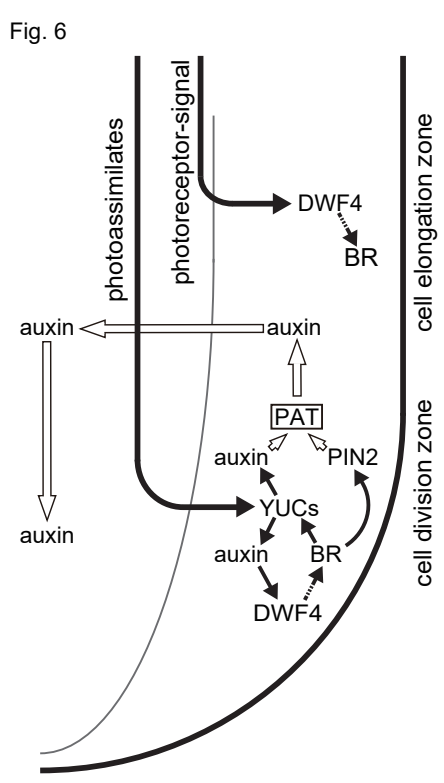

Fig. 6 | Model of the auxin-BR interaction in the root tip initiated by light-dependent signaling. The shoot tissue produces two different signals percieved by the primary root tip. In the cell elongation zone, the photoreceptor-derived signal promotes DWF4 accumulation and the subsequent BR biosynthesis. In the cell division zone, the photoassimilates promote the expression of several YUC genes. Additionally, auxin synthesized in the root tip promotes DWF4 expression and the subsequent BR biosynthesis. Next, BR induces the PIN2 localization in the PM. Moreover, BR contributes to the auxin flux into the stele because it establishes the PAT into the cell division zone.

It is likely that the PAT via PIN2 enhanced by BR signaling mediates this auxin flux. 